\title{
BIORTHOGONALITY IN THE BANACH SPACES $\ell^{p}(n)^{*}$
}

\author{
by ANTHONY J. FELTON and H. P. ROGOSINSKI
}

(Received 13th July 1994)

\begin{abstract}
We consider the finite-dimensional Banach spaces $\ell^{p}(n)$, where $p>1$. On these spaces there is a unique homogeneous semi-inner-product $[.,$.$] consistent with the norm. If p \neq 2$ this semi-inner product is not symmetric. We define a pair of vectors $x$ and $y$ to be biorthogonal if $[x, y]=[y, x]=0$. For a given non-zero $x$, let $\tau(\mathbf{x})$ be the number of elements in a maximal linearly independent set of vectors biorthogonal to $x$. If $p=2$ it is well-known that this number is $n-1$. The aim of this paper is to find $\tau(x)$ when $p \neq 2$. Our investigation shows that the situation differs from the Euclidean case in that the value of $\tau(x)$ can be either $n-1$ or $n-2$. The 'exceptional' vectors $\mathrm{x}$ for which $\tau(\mathbf{x})=n-2$ are characterised.
\end{abstract}

1991 Mathematics subject classification: $46 \mathrm{C} 50$.

\section{Introduction}

The following definition is due to Lumer [2]. Let $V$ be a real vector space. A semi-inner-product (s.i.p.) on $V \times V$ is a map $[.,]:. V \times V \rightarrow \mathbb{R}$ satisfying the following properties: for all $\mathbf{x}, \mathbf{y}, \mathbf{z} \in V$,

(a) $[\mathbf{x}+\mathbf{y}, \mathbf{z}]=[\mathbf{x}, \mathbf{z}]+[\mathbf{y}, \mathbf{z}]$,

(b) $[\lambda \mathbf{x}, \mathbf{y}]=\lambda[\mathbf{x}, \mathbf{y}] \forall \lambda \in \mathbb{R}$,

(c) $[x, x]>0$ if $x \neq 0$,

(d) $|[\mathbf{x}, \mathbf{y}]|^{2} \leqq[\mathbf{x}, \mathbf{x}][\mathbf{y}, \mathbf{y}]$.

We note in general $[\mathbf{x}, \mathbf{y}] \neq[\mathbf{y}, \mathbf{x}]$. A semi-inner-product is called homogeneous $[1]$ if

$$
[\mathbf{x}, \lambda \mathbf{y}]=\lambda[\mathbf{x}, \mathbf{y}] \forall \lambda \in \mathbb{R}, \text { and for all } \mathbf{x}, \mathbf{y} \in V \text {. }
$$

It is readily verified that $\|x\|=[x, x]^{1 / 2}$ defines a norm on $V$. We note the well-known result that in a smooth normed linear space $X$ there exists a unique semi-inner-product on $X$ which is consistent with the norm on $X$. In fact $[\mathbf{x}, \mathbf{y}]=(W \mathbf{y})(\mathbf{x})$ where $W \mathbf{y}$ is the unique linear functional such that $\|W \mathbf{y}\|=\|\mathbf{y}\|$ and $(W \mathbf{y})(\mathbf{x})=\|\mathbf{y}\|^{2}$. [2]

Definition 0.1. Let $X$ be a smooth normed linear space, with norm $\|$.$\| and$

*This research was funded by the University of Wales. 
associated semi-inner-product [.,.]. Let $\mathbf{x}, \mathbf{y} \in X$. We say that $\mathbf{x}$ is biorthogonal to $\mathbf{y}$ if, and only if,

$$
[\mathbf{y}, \mathbf{x}]=[\mathbf{x}, \mathbf{y}]=0
$$

In this case, we write $\mathbf{x} \pm \mathbf{y}$.

In the following we consider real finite-dimensional normed linear spaces $\ell^{p}(n)$, where $1<p<\infty, p \neq 2$. It is well-known that these spaces are smooth, and that the unique consistent s.i.p. on such spaces is given by

$$
[\mathbf{x}, \mathbf{y}]=\frac{1}{\|\mathbf{y}\|^{p-2}} \sum_{i=1}^{n} x_{i}\left|y_{i}\right|^{p-1} \operatorname{sgn} y_{i}=\frac{1}{\|\mathbf{y}\|^{p-2}} \sum_{i=1}^{n} x_{i} y_{i}\left|y_{i}\right|^{p-2}
$$

for $x, y \in \ell^{p}(n)$ and $y \neq 0$ (see e.g. [1]).

Throughout this paper $p$ will denote a real number such that $p>1$ and $p \neq 2$, Let $\mathbf{x}=\left(x_{1}, x_{2}, \ldots, x_{n}\right) \in \ell^{p}(n)$. We define $|\mathbf{x}|=\left(\left|x_{1}\right|,\left|x_{2}\right|, \ldots,\left|x_{n}\right|\right)$. Further we define $\mathbf{x}_{n}=$ $\left(x_{\pi(1)}, x_{\pi(2)}, \ldots, x_{\pi(n)}\right)$, where $\pi \in S_{n}$, and $S_{n}$ is the group of permutations of $\{1,2, \ldots, n\}$.

Definition 0.2. Let $\mathbf{x} \in \ell^{p}(n)$. Define $\tau(\mathbf{x})$ to be the number of elements in a maximally linearly independent set of vectors biorthogonal to $\mathbf{x}$.

Our purpose is to find $\tau(\mathbf{x})$ for each $\mathbf{x} \in \ell^{p}(n)$.

\section{Basic properties of $\tau(\mathbf{x})$}

Proposition 1.1. Let $\mathrm{x} \in \ell^{p}(n)$. For fixed $k \in \mathbb{N}$, let $\hat{\mathbf{x}} \in \ell^{p}(n+k)$ be defined by $\hat{\mathbf{x}}=(x \overbrace{0,0, \ldots, 0}^{k})$. Then

(i) $\tau(\lambda \mathbf{x})=\tau(\mathbf{x}) \forall \lambda \neq 0$,

(ii) $\tau(\mathbf{x})=\tau(|\mathbf{x}|)$,

(iii) $\tau(\mathbf{x})=\tau\left(\mathbf{x}_{\pi}\right)$ where $\pi \in S_{n}$,

(iv) $\tau(\hat{\mathbf{x}})=\tau(\mathbf{x})+k$,

(v) $\tau(\mathbf{x})=n$ if, and only if, $\mathbf{x}=\mathbf{0}$,

(vi) $n-2 \leqq \tau(\mathbf{x}) \leqq n$.

Proof. (i) Since

$$
[\lambda \mathbf{x}, \mathbf{y}]=\lambda[\mathbf{x}, \mathbf{y}],[\mathbf{y}, \lambda \mathbf{x}]=\lambda[\mathbf{y}, \mathbf{x}],
$$

$\mathbf{x} \pm \mathbf{y}$ if, and only if, $\lambda \mathbf{x} \pm \mathbf{y}$, when $\lambda \neq 0$ 
(ii) Define $\phi_{\mathbf{x}}: \ell^{p}(n) \rightarrow \ell^{p}(n)$ by

$$
\phi_{x}\left(\left(y_{1}, y_{2}, \ldots, y_{n}\right)\right)=\left(\left(\operatorname{sgn} x_{1}\right) y_{1},\left(\operatorname{sgn} x_{2}\right) y_{2}, \ldots,\left(\operatorname{sgn} x_{n}\right) y_{n}\right) \text {. }
$$

Then it is clear that the map $\phi_{\mathbf{x}}$ is linear and onto, and so preserves the linear dependence and linear independence of sets of vectors. Moreover the map $\phi_{\mathbf{x}}$ preserves the semi-inner-product on $\ell^{p}(n)$, and so

$$
\mathbf{y} \pm \mathbf{x} \text { if, and only if, } \phi_{\mathbf{x}}(\mathbf{y}) \pm \phi_{\mathbf{x}}(\mathbf{x})
$$

Since $\phi_{\mathbf{x}}(\mathbf{x})=|\mathbf{x}|$, it follows that $\tau(\mathbf{x})=\tau(|\mathbf{x}|)$.

(iii) The proof of this follows from the identity

$$
\sum_{i=1}^{n} x_{i} y_{i}\left|y_{i}\right|^{p-2}=\sum_{i=1}^{n} x_{\pi(i)} y_{\pi(i)}\left|y_{\pi(i)}\right|^{p-2}
$$

where $\pi \in S_{n}$, as well as from the fact that the map $\mathbf{x} \rightarrow \mathbf{x}_{\pi}$ preserves linear independence.

(iv) Note that $\left(y_{1}, \ldots, y_{n+k}\right)$ is biorthogonal to $\hat{\mathbf{x}}$ in $\ell^{p}(n+k)$ if, and only if, $\left(y_{1}, \ldots, y_{n}\right)$ is biorthogonal to $\mathbf{x}$ in $\ell^{p}(n)$. If $\mathbf{e}_{1}, \ldots, \mathbf{e}_{n+k}$ are the standard basis vectors in $\ell^{p}(n+k)$, and if $b_{1}, \ldots, b_{\tau(x)}$ is a set of $\tau(\mathbf{x})$ linearly independent vectors biorthogonal to $\mathbf{x}$, it follows that $\left\{\hat{\mathbf{b}}_{1}, \hat{\mathbf{b}}_{2}, \ldots, \hat{\mathbf{b}}_{\mathbf{r}(x)}, \mathbf{e}_{n+1}, \ldots, \mathbf{e}_{n+k}\right\}$ is a set of $\tau(\mathbf{x})+k$ linearly independent vectors biorthogonal to $\hat{\mathbf{x}}$ in $\ell^{p}(n+k)$. Moreover every vector $\left(y, \ldots, y_{n+k}\right)$ biorthogonal to $\hat{\mathbf{x}}$ is in the linear span of these $\tau(\mathbf{x})+k$ vectors. Indeed, since $\left(y_{1}, \ldots, y_{n}\right)$ is biorthogonal to $\mathbf{x}$ there exist scalars $\lambda_{1}, \ldots, \lambda_{r(x)}$ so that

$$
\left(y_{1}, \ldots, y_{n}\right)=\lambda_{1} \mathbf{b}_{1}+\lambda_{2} \mathbf{b}_{2}+\ldots+\lambda_{\tau(\mathbf{x})} \mathbf{b}_{\tau(\mathbf{x})} \text {, }
$$

and so

$$
\left(y_{1}, \ldots, y_{n+k}\right)=\lambda_{1} \hat{\mathbf{b}}_{1}+\lambda_{2} \hat{\mathbf{b}}_{2}+\ldots+\lambda_{\tau(\mathbf{x})} \hat{\mathbf{b}}_{\tau(\mathbf{x})}+y_{n+1} \mathbf{e}_{n+1}+\ldots+y_{n+k} \mathbf{e}_{n+k} .
$$

This completes the proof that $\tau(\hat{\mathbf{x}})=\tau(\mathbf{x})+k$.

(v) If $\tau(\mathbf{x})=n$ then there exists a basis in $\ell^{p}(n)$ in which each vector is biorthogonal to $x$. Since the semi-inner-product in $\ell^{p}(n)$ is left-linear, it follows that all vectors in $\ell^{p}(n)$ are left-orthogonal to $\mathbf{x}$. In particular $[\mathbf{x}, \mathbf{x}]=0$, and so $\mathbf{x}=\mathbf{0}$.

(vi) We need only show that $\tau(x) \geqq n-2$ whenever $x \in \ell^{p}(n)$. This is obvious when $n=2$. We proceed by induction. Fix $k$, with $k \geqq 2$, and assume that $\tau(\mathbf{x}) \geqq k-2$ whenever $\mathbf{x} \in \ell^{p}(k)$. Let $\mathbf{x}=\left(x_{1}, x_{2}, \ldots, x_{k+1}\right) \in \ell^{p}(k+1)$. We shall show in Section 3 (Proposition 3.3(i)) that there exists a non-zero vector $\left(b_{k-1}, b_{k}, b_{k+1}\right)$ biorthogonal to $\left(x_{k-1}, x_{k}, x_{k+1}\right)$. Let $\mathbf{b}_{1}$ be the vector in $\ell^{p}(k+1)$ given by

$$
b_{1}=\overbrace{\left(0, \ldots, 0, b_{k-1}, b_{k}, b_{k+1}\right)}
$$

Then $\mathbf{b}_{1}$ is biorthogonal to $\mathbf{x}$. We shall assume that $b_{k+1} \neq 0$ (If $b_{k+1}=0$ then either $b_{k-1}$ 
or $b_{k}$ must be non-zero, and it is clear how to modify the argument which follows). By the inductive assumption there exists a set of $k-2$ linearly independent vectors biorthogonal to $\left(x_{1}, x_{2}, \ldots, x_{k}\right) \in \ell^{p}(k)$. Let $\mathbf{b}_{2}, \ldots, \mathbf{b}_{k-1}$ be the $k-2$ vectors in $\ell^{p}(k+1)$ arising from this set by the addition of a final coordinate which is equal to 0 . Then each of these 'augmented' vectors is biorthogonal to $x$. Moreover, since the final coordinate of $\mathbf{b}_{1}$ is non-zero, the set of vectors $\left\{\mathbf{b}_{1}, \mathbf{b}_{2}, \ldots, \mathbf{b}_{k-1}\right\}$ is linearly independent, and it follows that $\tau(x) \geqq k-1$. This completes the proof.

\section{Biorthogonality in $\ell^{p}(2)$}

Proposition 2.1. Let $\mathrm{x}=(a, b) \in \ell^{p}(2)$. Then

(i) $\tau(\mathbf{x})=2$ if, and only if, $\mathbf{x}=\mathbf{0}$.

(ii) If either $a$ or $b$ is equal to zero, and if $\mathbf{x} \neq \mathbf{0}$, then $\tau(\mathbf{x})=1$.

(iii) If both $a$ and $b$ are non-zero then $\tau(\mathbf{x})=1$ if, and only if, $|a|=|b|$.

Proof. (i) This is covered by Proposition 1.1(v).

(ii) If $a=0$ then $(1,0)$ is biorthogonal to $\mathbf{x}$, and if $b=0$ then $(0,1)$ is biorthogonal to $\mathbf{x}$. Since $\mathbf{x} \neq \mathbf{0}$, it follows that $\tau(\mathbf{x})=1$.

(iii) If $a=b$ then $(1,-1)$ is biorthogonal to $\mathbf{x}$, and if $a=-b$ then $(1,1)$ is biorthogonal to $\mathbf{x}$. In either case since $\mathbf{x} \neq \mathbf{0}, \tau(\mathbf{x})=1$. Suppose conversely that $\tau(\mathbf{x})=1$. Then there exists a non-zero vector $(c, d)$ biorthogonal to $(a, b)$. Since $b \neq 0$ it follows that $c \neq 0$. Since the s.i.p. is homogeneous we can assume w.l.o.g. that $c=1$. We then have

$$
a|a|^{p-2}+d b|b|^{p-2}=0 \text { and } \quad a+b d|d|^{p-2}=0 .
$$

The first equation implies that

$$
|a|^{p-1}=|d||b|^{p-1}
$$

whilst the second equation implies that

$$
|a|=|b||d|^{p-1}
$$

Substituting for $|d|^{p-1}$ from (2) into (1) gives

$$
|a|^{(p-1)^{2}}=\frac{|a|}{|b|}|b|^{(p-1)^{2}} .
$$

Hence $|a|^{p(p-2)}=|b|^{p(p-2)}$, and since $p \neq 2$ it follows that $|a|=|b|$.

\section{Biorthogonality in $\ell^{p}(3)$}

We start with a lemma.

Lemma 3.1. Let $p>1$ with $p \neq 2$, and let $a \geqq b \geqq 1$. Let $f$ be defined on $(-\infty, \infty)$ by 


$$
f(t)=a+b t|t|^{p-2}-\left[a^{p-1}+b^{p-1} t\right]\left|a^{p-1}+b^{p-1} t\right|^{p-2} .
$$

\section{Then}

(i) if $b>1, f$ has at least one zero, and $f$ has more than one zero if, and only if, $a^{p} \leqq b^{p}+1$

(ii) if $b=1, f$ has a zero if, and only if, $a^{p} \leqq 2$.

(iii) $(1, x, y)$ is biorthogonal to $(a, b, 1)$ in $\ell^{p}(3)$ if, and only if, $f(x)=0$ and $y=$ $-\left[a^{p-1}+b^{p-1} x\right]$.

Proof. We shall assume throughout that $p>2$. The case where $1<p<2$ is treated similarly.

(i) Let $b>1$. Note that

$$
\lim _{|x| \rightarrow \infty} \frac{f(t)}{t|t|^{p-2}}=b\left[1-b^{p(p-2)}\right]<0
$$

It follows that

$$
\lim _{t \rightarrow-\infty} f(t)=\infty \text { and } \lim _{t \rightarrow \infty} f(t)=-\infty .
$$

Since $f$ is continuous, the intermediate-value theorem shows that $f$ has a zero on $(-\infty, \infty)$. By elementary calculus

$$
f^{\prime}(t)=(p-1)\left[b|t|^{p-2}-b^{p-1}\left|a^{p-1}+b^{p-1} t\right|^{p-2}\right] .
$$

Let

$$
t_{0}=\frac{-a^{p-1} b}{b^{p}-1} \text { and } t_{1}=\frac{-a^{p-1} b}{b^{p}+1}
$$

Then it is easily verified that

$$
f^{\prime}\left(t_{0}\right)=f^{\prime}\left(t_{1}\right)=0, f^{\prime}(t)>0 \quad \text { if } \quad t_{0}<t<t_{1} \text {, and } f^{\prime}(t)<0 \text { otherwise. }
$$

Hence $f$ attains a minimum value when $t=t_{0}$ and a maximum value when $t=t_{1}$. Since $a^{p}>b^{p}-1$ and $p>2$,

$$
f\left(t_{0}\right)=\frac{a}{\left(b^{p}-1\right)^{p-2}}\left[\left(b^{p}-1\right)^{p-2}-a^{p(p-2)}\right]<0 .
$$

It is now clear that $f\left(t_{1}\right) \geqq 0$ is a necessary and sufficient condition for $f$ to have more than one zero. Since

$$
f\left(t_{1}\right)=\frac{a}{\left(b^{p}+1\right)^{p-2}}\left[\left(b^{p}+1\right)^{p-2}-a^{p(p-2)}\right]
$$


this condition is equivalent to $a^{p} \leqq b^{p}+1$.

(ii) Let $b=1$. Then, putting $c=a^{p-1}$,

$$
f(t)=a+t|t|^{p-2}-(c+t)|c+t|^{p-2} .
$$

We have

$$
f^{\prime}(t)=(p-1)\left[|t|^{p-2}-|c+t|^{p-2}\right],
$$

and so $f^{\prime}(t)>0$ if $|t|>|c+t|$ and $f^{\prime}(t)<0$ if $|t|<|c+t|$. Hence $f^{\prime}(t)>0$ if $t<-\frac{1}{2} c$ and $f^{\prime}(t)<0$ if $t>-\frac{1}{2} c$, and consequently $f$ attains its maximum value when $t=-\frac{1}{2} c$. This maximum value is given by

$$
f\left(-\frac{1}{2} c\right)=a-\frac{c^{p-1}}{2^{p-2}}=\frac{a}{2^{p-2}}\left(2^{p-2}-a^{p(p-2)}\right) .
$$

If $a^{p}>2$ then this maximum value is negative, and so $f$ has no zeros. Otherwise, $f\left(-\frac{1}{2} c\right) \geqq 0$ whereas $f(0)=a-c^{p-1}=a-a^{(p-1)^{2}} \leqq 0$, and by the intermediate-value theorem, $f$ has a zero in the closed interval $\left[-\frac{1}{2} c, 0\right]$.

(iii) $(1, x, y)$ is biorthogonal to $(a, b, 1)$ if, and only if,

$$
a+b x|x|^{p-2}+y|y|^{p-2}=0,
$$

and

$$
a^{p-1}+b^{p-1} x+y=0 .
$$

This is clearly the case if, and only if,

$$
y=-\left[a^{p-1}+b^{p-1} x\right],
$$

and

$$
f(x)=a+b x|x|^{p-2}-\left[a^{p-1}+b^{p-1} x\right]\left|a^{p-1}+b^{p-1} x\right|^{p-2}=0 .
$$

Corollary 3.2. Let $\mathbf{x}=(a, b, 1) \in \ell^{p}(3)$ where $a>b>1$. Then there exist real numbers $\alpha, \alpha^{\prime}$ with $\alpha<0$ and $\alpha^{\prime}>0$ such that $\left(1, \alpha, \alpha^{\prime}\right)$ is biorthogonal to $\mathbf{x}$.

Proof. Let $p>2$. The case $1<p<2$ is treated similarly. Let $f$ be the function defined in Lemma 3.1. Since $b>1$, we have seen that $f(t) \rightarrow \infty$ as $t \rightarrow-\infty$. Since

$$
f\left(-\left(\frac{a}{b}\right)^{p-1}\right)=\frac{a}{b^{p(p-2)}}\left[b^{p(p-2)}-a^{p(p-2)}\right]<0 .
$$

it follows that $f(\alpha)=0$ for some $\alpha$, with $\alpha<-\left(\frac{a}{b}\right)^{p-1}<0$. If $\alpha^{\prime}=-\left[a^{p-1}+b^{p-1} \alpha\right]$, then $\alpha^{\prime}>0$. By Lemma 3.1(iii), $\left(1, \alpha, \alpha^{\prime}\right)$ is biorthogonal to $\mathbf{x}$. 
Proposition 3.3. Let $p>1, p \neq 2$. Let $\mathrm{x}=(a, b, c) \in \ell^{p}(3)$. Then

(i) $\tau(\mathbf{x}) \geqq 1$,

(ii) If $a, b, c$ are non-zero, and if $\{\alpha, \beta, \gamma\}$ is a permutation of $\{a, b, c\}$ with $|\alpha| \geqq|\beta| \geqq|\gamma|$, then $\tau(\mathbf{x})=2$ if, and only if, $|\alpha|^{p} \leqq|\beta|^{p}+|\gamma| p$.

Proof. In what follows $f$ is the function defined in Lemma 3.1.

(i) Let $\mathrm{x}=(a, b, c)$. We can assume that $a, b, c$ are non-zero, since otherwise one of the vectors $(1,0,0),(0,1,0),(0,0,1)$ will be biorthogonal to $\mathbf{x}$. By Proposition 1.1(i), (ii), and (iii), we can assume w.l.o.g. that $a \geqq b \geqq c=1$. Moreover if $b=c$ then $(0,1,-1)$ is biorthogonal to $x$, and so we can further assume that $a \geqq b>c=1$. Lemma 3.1(i) then shows that $f(x)=0$ for some real $x$, and so, by Lemma 3.1(iii), $(1, x, y)$ is biorthogonal to $\mathrm{x}$, where $y=-\left[a^{p-1}+b^{p-1} x\right]$. Hence $\tau(\mathbf{x}) \geqq 1$.

(ii) Let $\mathbf{x}=(a, b, c)$ where $a, b, c$ are non-zero. Again we may assume that $a \geqq b \geqq c=1$. We consider two cases.

Case 1. Let $b>1$. By Proposition 2.1(iii), $\tau(b, 1)=0$, and consequently there is no non-zero vector of the form $(0, x, y)$ biorthogonal to $\mathbf{x}$. Hence $\tau(\mathbf{x})=2$ if, and only if, there are two linearly independent vectors of the form $(1, x, y)$ biorthogonal to $x$. Lemma 3.1(iii) shows that this happens if, and only if, the function $f$ has more than one zero, and so if, and only if, $a^{p} \leqq b^{p}+1=b^{p}+c^{p}$ (Lemma 3.1(i)).

Case 2. Let $b=1$. Then $x=(a, 1,1)$. Since $\tau(1,1)=1$, the only vectors of the form $(0, x, y)$ biorthogonal to $\mathrm{x}$ are scalar multiples of $(0,1,-1)$. Hence $\tau(\mathbf{x})=2$ if, and only if, there is some vector $(1, x, y)$ biorthogonal to $x$. Lemma 3.1(iii) shows that this happens if, and only if, $f(x)=0$ for some $x$, and so if, and only if, $a^{p} \leqq 2=b^{p}+c^{p}$ (Lemma 3.1(ii)).

Corollary 3.4. Let $n \geqq 3$. Let $\mathrm{x}=\left(x_{1}, x_{2}, \ldots, x_{n}\right) \in \ell^{p}(n)$ with $x_{1} \geqq x_{2} \geqq \ldots \geqq x_{n}>0$. Then there exists a vector $\mathbf{y}$ of the form $\left(y_{1}, y_{2}, \ldots, y_{n-1}, 1\right)$ which is biorthogonal to $\mathbf{x}$.

Proof. By putting $y_{1}=y_{2}=\ldots=y_{n-3}=0$ we may assume w.l.o.g. that $n=3$. Let $\mathrm{x}=\left(x_{1}, x_{2}, x_{3}\right)$ with $x_{1} \geqq x_{2} \geqq x_{3}>0$. If $x_{1}>x_{2}$ the result follows from the facts that $\tau\left(x_{1}, x_{2}\right)=0$ (Proposition 2.1(iii)) and $\tau\left(x_{1}, x_{2}, x_{3}\right) \geqq 1$ (Proposition 3.3(i)). If $x_{1}=x_{2}$ the result follows from the fact that $\tau\left(x_{1}, x_{2}\right)=1$ (Proposition 2.1(iii) and $\tau\left(x_{1}, x_{2}, x_{3}\right)=2$ (Proposition 3.3(ii)).

\section{The main theorem}

The proof of the following proposition makes use of an inequality which we state in the form of a preliminary lemma. We recall that throughout $p>1$ and $p \neq 2$.

Lemma 4.1. If $b \geqq c \geqq 1$, and $\lambda>0$ then

$$
\left(b+c \lambda^{p-1}\right)-\left(b^{p-1}+\lambda c^{p-1}\right)^{p-1} \neq 0
$$


Proof. Suppose that $p>2$. Elementary calculus shows that $\left(x_{1}+x_{2}\right)^{p-1}>x_{1}^{p-1}+x_{2}^{p-1}$ when $x_{1}, x_{2}>0$. With $x_{1}=b^{p-1}, x_{2}=\lambda c^{p-1}$ this inequality reduces to $\left(b^{p-1}+\lambda c^{p-1}\right)^{p-1}>b^{(p-1)^{2}}+\lambda^{p-1} c^{(p-1)^{2}}$. Since $b^{(p-1)^{2}} \geqq b$ and $c^{(p-1)^{2}} \geqq c$, we see that the expression on the left-hand side of (1) is negative. A similar argument shows that this expression is positive if $1<p<2$.

Proposition 4.2. Let $a \geqq b \geqq c \geqq 1$. Let $\mathrm{x}=(a, b, c, 1) \in \ell^{p}(4)$. Then for each $\lambda>0$ there exist $x_{0}(\lambda)$ and $z_{0}(\lambda)$ such that $\left(1, x_{0}(\lambda), \lambda x_{0}(\lambda), z_{0}(\lambda)\right)$ is biorthogonal to $\mathbf{x}$. Moreover if $a>b \geqq c=1$ then $x_{0}(\lambda)$ is not constant on $(0, \infty)$.

Proof. Let $\lambda>0$ and let $\mathbf{y}=\left(1, t, \lambda t, t^{\prime}\right) \in \ell^{p}(4)$. Then $\mathbf{x}$ is biorthogonal to $\mathbf{y}$ if, and only if,

and

$$
a+\left(b+c \lambda^{p-1}\right) t|t|^{p-2}+t^{\prime}\left|t^{\prime}\right|^{p-2}=0
$$

$$
a^{p-1}+\left(b^{p-1}+\lambda c^{p-1}\right) t+t^{\prime}=0
$$

Substituting (2) into (1) we obtain the equation

$$
f_{\lambda}(t)=a+\left(b+c \lambda^{p-1}\right) t|t|^{p-2}-\left(a^{p-1}+\left(b^{p-1}+\lambda c^{p-1}\right) t\right)\left|a^{p-1}+\left(b^{p-1}+\lambda c^{p-1}\right) t\right|^{p-2}=0 .
$$

We have

$$
\frac{f_{\lambda}(t)}{t|t|^{p-2}} \rightarrow\left(b+c \lambda^{p-1}\right)-\left(b^{p-1}+\lambda c^{p-1}\right)^{p-1}, \quad \text { as }|t| \rightarrow \infty
$$

By Lemma 4.1 this limit is non-zero, and it then follows from the intermediate-value theorem that equation (3) has a real root. For $\lambda>0$, let $x_{0}(\lambda)$ denote the least such root.

If

$$
z_{0}(\lambda)=-\left[a^{p-1}+\left(b^{p-1}+\lambda c^{p-1}\right) x_{0}(\lambda)\right]
$$

it is clear that $\left(1, x_{0}(\lambda), \lambda x_{0}(\lambda), z_{0}(\lambda)\right)$ is biorthogonal to $x$.

Let $a>b \geqq c=1$. Suppose, for a contradiction, that for some constant $K, x_{0}(\lambda)=K$ for all positive $\lambda$. Then

$$
f_{\lambda}(K)=a+\left(b+\lambda^{p-1}\right) K|K|^{p-2}-\left(a^{p-1}+\left(b^{p-1}+\lambda\right) K\right)\left|a^{p-1}+\left(b^{p-1}+\lambda\right) K\right|^{p-2}=0 \forall \lambda>0 .
$$

Since $a>1$, we see from (4) that $K \neq 0$. Differentiating both sides of (4) with respect to $\lambda$ we obtain

$$
\left.(p-1) \lambda^{p-2} K|K|^{p-2}-\mid a^{p-1}+\left(b^{p-1}+\lambda\right) K\right)\left.\right|^{p-2}(p-1) K=0 \forall \lambda>0 .
$$


Dividing both sides of (5) by $(p-1) K|K|^{p-2}$ and taking $(p-2)^{t h}$ roots gives

$$
\lambda=\left|\frac{a^{p-1}}{K}+b^{p-1}+\lambda\right| \forall \lambda>0,
$$

and (6) implies that

$$
K=-\frac{a^{p-1}}{b^{p-1}}
$$

Since

$$
f_{\lambda}\left(\frac{-a^{p-1}}{b^{p-1}}\right)=\frac{a\left(b^{p(p-2)}-a^{p(p-2)}\right)}{b^{p(p-2)}} \neq 0
$$

we obtain the desired contradiction.

Proposition 4.3. Let $\mathbf{x}=(a, b, c, d) \in \ell^{p}(4)$, where $a, b, c, d$ are non-zero. Then $\tau(\mathbf{x})=3$.

Proof. By (i), (ii), (iii) of Proposition 1.1, we can assume w.l.o.g. that $a \geqq b \geqq c \geqq d=1$. We consider three cases.

Case 1. Suppose that at least two of $a, b, c$ are equal.

Suppose first that $a=b$. Then $\tau(a, b, c)=2$ by Proposition 3.3(ii). Hence there are two linearly independent vectors in $\ell^{p}(4)$ which are biorthogonal to $(a, b, c, 1)$ and whose last coordinates are 0 . By Corollary 3.4 there is also a vector in $\ell^{p}(4)$ which is biorthogonal to $(a, b, c, 1)$ and whose last coordinate is 1 . The three vectors so obtained are linearly independent, and hence $\tau(a, b, c, 1)=3$.

Now suppose that $b=c$. Then $\tau(b, c, 1)=2$ by Proposition 3.3(ii). Hence there are two linearly independent vectors in $\ell^{p}(4)$ which are biorthogonal to $(a, b, c, 1)$ and whose first coordinates are 0 . By Proposition 4.2, there is also a vector in $\ell^{p}(4)$ which is biorthogonal to $(a, b, c, 1)$ whose first coordinate is 1 . Again $\tau(a, b, c, 1)=3$.

Case 2. Suppose that $a>b>c>1$.

Corollary 3.2 shows that there exist vectors $\mathbf{X}=\left(1,0, \alpha, \alpha^{\prime}\right), \quad \mathbf{Y}=\left(0,1, \beta, \beta^{\prime}\right)$, $\mathbf{Z}=\left(1, \gamma, 0, \gamma^{\prime}\right)$ biorthogonal to $\mathbf{x}$, where $\alpha, \beta, \gamma<0$ and $\alpha^{\prime}, \beta^{\prime}, \gamma^{\prime}>0$. We shall show that the vectors $X, Y, Z$ are linearly independent, by showing that

$$
\left|\begin{array}{lll}
1 & 0 & \alpha \\
0 & 1 & \beta \\
1 & \gamma & 0
\end{array}\right| \neq 0
$$

In fact suppose, for a contradiction, that this determinant is zero. Then 


$$
\beta=-\frac{\alpha}{\gamma}
$$

Since $[\mathbf{X}, \mathbf{X}]=[\mathbf{Y}, \mathbf{X}]=[\mathbf{Z}, \mathbf{X}]=0$, we have

$$
\alpha^{\prime}=-\left(a^{p-1}+c^{p-1} \alpha\right), \quad \beta^{\prime}=-\left(b^{p-1}+c^{p-1} \beta\right), \quad \gamma^{\prime}=-\left(a^{p-1}+b^{p-1} \gamma\right)
$$

and it follows from (1) and (2) that

$$
\frac{\gamma^{\prime}-\alpha^{\prime}}{\gamma}=\beta^{\prime}
$$

Since $\gamma<0$ and $\beta^{\prime}>0$, we see that $\alpha^{\prime}>\gamma^{\prime}$.

By (1) and (3),

$$
-\gamma \mathbf{Y}=\left(0,-\gamma,-\gamma \beta,-\gamma \beta^{\prime}\right)=\left(0,-\gamma, \alpha, \alpha^{\prime}-\gamma^{\prime}\right),
$$

and since $[\mathbf{x},-\gamma \mathbf{Y}]=0$ we have, noting that $\alpha^{\prime}>\gamma^{\prime}$,

$$
-b \gamma|\gamma|^{p-2}+c \alpha|\alpha|^{p-2}+\left(\alpha^{\prime}-\gamma^{\prime}\right)^{p-1}=0
$$

Now $[x, X]=[x, Z]=0$, and so we also have

$$
[\mathbf{X}, \mathbf{X}]-[\mathbf{X}, \mathbf{Z}]=-b \gamma|\gamma|^{p-2}+c \alpha|\alpha|^{p-2}+\alpha^{p-1}-\gamma^{\prime p-1}=0 .
$$

Subtracting (5) from (4), we deduce that

$$
\left(\alpha^{\prime}-\gamma^{\prime}\right)^{p-1}-\alpha^{\prime p-1}+\gamma^{p-1}=0
$$

If

$$
r=\frac{\alpha^{\prime}}{\gamma^{\prime}}
$$

this gives

$$
(r-1)^{p-1}-r^{p-1}+1=0 .
$$

Elementary calculus shows that, since $p \neq 2$, the expression on the left-hand side of $(6)$ is strictly monotonic in $r$, and so (6) is satisfied only when $r=1$. Since

$$
r=\frac{\alpha^{\prime}}{\gamma^{\prime}}>1
$$

we have the desired contradiction.

Case 3. Suppose that $a>b>c=1$. 
If $y_{1}=(0,0,1,-1)$, then $y_{1}$ is biorthogonal to $x$. Corollary 3.2 shows that there exists a vector $y_{2}=\left(1, \alpha, 0, \alpha^{\prime}\right)$ biorthogonal to $x$. By Proposition 4.2 for each $\lambda>0$ there exist $x(\lambda)$ and $z(\lambda)$ such that $\mathbf{y}_{3}(\lambda)$ is biorthogonal to $\mathbf{x}$, where $\mathbf{y}_{3}(\lambda)=(1, x(\lambda), \lambda x(\lambda), z(\lambda))$. Suppose, for a contradiction, that the three vectors $\mathbf{y}_{1}, \mathbf{y}_{2}, \mathbf{y}_{3}(\lambda)$ are linearly dependent for all $\lambda>0$. Then

$$
\left|\begin{array}{ccc}
0 & 0 & 1 \\
1 & \alpha & 0 \\
1 & x(\gamma) & \lambda x(\lambda)
\end{array}\right| \neq 0
$$

This implies that

$$
x(\lambda)=\alpha \forall \lambda>0
$$

and so contradicts the second part of Proposition 4.2. Hence $\tau(\mathbf{x})=3$.

Proposition 4.4. Let $n \geqq 4$ and let $\mathrm{x}=\left(x_{1}, x_{2}, \ldots, x_{n}\right) \in \ell^{p}(n)$ where $x_{i} \neq 0 \forall i$. Then

$$
\tau(\mathbf{x})=n-1
$$

Proof. We proceed by induction. Proposition 4.3 shows that (1) holds when $n=4$. Let $k \geqq 4$, and suppose that (1) holds when $n=k$. Let $\mathbf{x}=\left(x_{1}, x_{1}, \ldots, x_{k+1}\right) \in \ell^{p}(k+1)$. By Proposition 1.1(ii), (iii) we may assume without loss of generality that $x_{1} \geqq x_{2} \geqq \ldots \geqq$ $x_{k+1}>0$. Then $\left(x_{1}, x_{2}, \ldots, x_{k}\right) \in \ell^{p}(k)$, and by the inductive hypothesis there exists a linearly independent set of $k-1$ vectors in $\ell^{p}(k)$ biorthogonal to $\left(x_{1}, x_{1}, \ldots, x_{k}\right)$. By adding a final zero coordinate to each of the vectors in this set, we obtain a linearly independent set of $k-1$ vectors $\mathbf{y}_{1}, \mathbf{y}_{2}, \ldots, \mathbf{y}_{k-1}$ in $\ell^{p}(k+1)$ biorthogonal to $\mathbf{x}$. By Corollary 3.4 there exists a vector $y_{k}$ with final coordinate equal to 1 which is biorthogonal to $\mathbf{x}$. The set of vectors $\left\{\mathbf{y}_{1}, \mathbf{y}_{2}, \ldots, \mathbf{y}_{k}\right\}$ is then a linearly independent set of $k$ vectors in $\ell^{p}(k+1)$ biorthogonal to $x$. Since $\mathbf{x} \neq 0, \tau(\mathbf{x})=k$. Hence (1) holds for $n=k+1$, and the proof is complete.

An application of Proposition 2.1(ii), (iii), Proposition 3.3(ii) and Proposition 4.4, together with the properties (ii), (iii), (iv) and (v) of $\tau(\mathbf{x})$ listed in Proposition 1.1 now readily yield our main result.

Theorem 4.5. Let $n \geqq 2$, and let $\mathrm{x} \in \ell^{p}(n)$. Let $k$ be the number of non-zero coordinates of $\mathbf{x}$.

(i) If $k=0$ then $\tau(\mathbf{x})=n$.

(ii) If $k=1$ or $k \geqq 4$ then $t(x)=n-1$.

(iii) If $k=2$ then $\tau(\mathbf{x})=n-1$ if the two non-zero coordinates have equal modulus, and $\tau(\mathbf{x})=n-2$ otherwise. 
(iv) If $k=3$, let $\{\alpha, \beta, \gamma\}$ be a permutation of the three non-zero coordinates such that $|\alpha| \geqq|\beta| \geqq|\gamma|$. Then $\tau(\mathbf{x})=n-1$ if $|\alpha|^{p} \leqq|\beta|^{p}+|\gamma|^{p}$ and $\tau(\mathbf{x})=n-2$ otherwise.

Acknowledgement. We would like to thank the referee for his helpful comments concerning the presentation of this paper.

\section{REFERENCES}

1. J. R. Giles, Classes of semi-inner-product spaces, Trans. Amer. Math. Soc. 129 (1967), 436-446.

2. G. Lumer, Semi-inner-product spaces, Trans. Amer. Math. Soc. 100 (1961), 29-43.

Department of Mathematics

University OF Wales, SWANSEa

Singleton Park

SWANSEa SA2 8PP

WALES 